\title{
Martensitic transitions and the nature of ferromagnetism in the austenitic and martensitic states of $\mathrm{Ni}-\mathrm{Mn}-\mathrm{Sn}$ alloys
}

\author{
Thorsten Krenke, Mehmet Acet,* and Eberhard F. Wassermann \\ Fachbereich Physik, Experimentalphysik, Universität Duisburg-Essen, D-47048 Duisburg, Germany \\ Xavier Moya, Lluís Mañosa, and Antoni Planes \\ Facultat de Física, Departament d'Estructura i Constituents de la Materìa, Universitat de Barcelona, Diagonal 647, \\ E-08028 Barcelona, Catalonia, Spain
}

(Received 24 November 2004; revised manuscript received 1 February 2005; published 5 July 2005)

\begin{abstract}
Structural and magnetic transformations in the Heusler-based system $\mathrm{Ni}_{0.50} \mathrm{Mn}_{0.50-x} \mathrm{Sn}_{x}$ are studied by $\mathrm{x}$-ray diffraction, optical microscopy, differential scanning calorimetry, and magnetization. The structural transformations are of austenitic-martensitic character. The austenite state has an $L 2_{1}$ structure, whereas the structures of the martensite can be $10 M, 14 M$, or $L 1_{0}$ depending on the Sn composition. For samples that undergo martensitic transformations below and around room temperature, it is observed that the magnetic exchange in both parent and product phases is ferromagnetic, but the ferromagnetic exchange, characteristic of each phase, is found to be of different strength. This gives rise to different Curie temperatures for the austenitic and martensitic states.
\end{abstract}

DOI: 10.1103/PhysRevB.72.014412

PACS number(s): 75.50.Cc, 81.30.Kf

\section{INTRODUCTION}

The martensitic transformation is a first-order solid-solid phase transition, which takes place by the diffusionless shearing of the parent austenitic phase. The main characteristics of a first-order martensitic phase transformation are the presence of supercooled and superheated states that lead to a thermal hysteresis of width $\Delta T$. The characteristic temperatures related to the martensitic transformation are the martensite start temperature $M_{s}$, martensite finish temperature $M_{f}$, austenite start temperature $A_{s}$, and the austenite finish temperature $A_{f}$. The martensitic transformation has broad applications, among which steel hardening and shape memory devices are of prime importance. One understands under the so-called shape memory effect the capability of a material to remember its original shape when it is deformed in the martensitic state and then heated to retransform to the austenitic state. One of the earliest known shape memory alloys is $\mathrm{Au}_{52.5} \mathrm{Cd}_{47.5}{ }^{1}$ Since then, many other systems were found, among which $\mathrm{Ni}$ - Ti has the widest application.

The austenite-martensite transformation in shape memory alloys is thermoelastic, which is a property characterized by a narrow thermal hysteresis of several ten Kelvins or less. This implies that the dissipative contribution to the free energy during the transformation is small, so that the deformation energy is essentially elastically stored in the twin morphology of the martensite structure. ${ }^{2}$ The two components of the twin have the same crystal structure, but different orientations, and are known as variants. The variants build selfaccommodating groups with other twins such that they minimize the elastic contribution to the free energy.

Some Heusler based alloys, such as $\mathrm{Cu}-\mathrm{Ni}-\mathrm{Al}, \mathrm{Cu}-\mathrm{Zn}-$ $\mathrm{Al}$, etc., also exhibit the shape memory effect. The stoichiometric Heusler compound $X_{2} Y Z$ crystallizes in the $L 2_{1}$ structure (space group $F m 3 m$ ), which is made up of four interpenetrating fcc sublattices. Each sublattice is occupied by one sort of atom. If the alloy transforms martensitically, the structure at $T<M_{s}$ usually has a modulated structure.

In the case when the Heusler alloy is magnetic, it can exhibit the magnetic shape memory (MSM) effect. This occurs especially when the $Y$ species is Mn, but other transition elements are also possible. In these alloys, an external magnetic field can induce large strains when applied in the martensitic state. ${ }^{3,4} \mathrm{In} \mathrm{Ni}_{2} \mathrm{MnGa}$, these strains can be as large as $10 \%$ in a field of about $1 \mathrm{~T}$. The field-induced strain is due to the reorientation of the tetragonal martensite variants by twin boundary motion. The driving force for the reorientation is provided by the difference in the Zeeman energy $\Delta \mathbf{M} \cdot \mathbf{H}$ of neighboring variants. ${ }^{5}$ The maximum achievable strain $\epsilon_{\max }$ depends on the tetragonality $c / a$ of the martensitic phase. ${ }^{6}$ Depending on the composition of the Ni-Mn-Ga alloy, the martensitic phase occurs as various modulated phases such as $10 M, 14 M$, or $L 1_{0}{ }^{7}$ Although Ni-Mn-Ga alloys offer large magnetic field-induced strains, they are brittle which makes it difficult for applications. Therefore, in the past few years, there has been more research invested in the search for materials exhibiting the MSM effect and having more favorable mechanical properties at the same time. Over the past few years, other MSM alloys, such as $\mathrm{Fe}-\mathrm{Pt},{ }^{8} \mathrm{Fe}-\mathrm{Pd},{ }^{9} \mathrm{Co}-$ $\mathrm{Ni}-\mathrm{Al},{ }^{10} \mathrm{Co}-\mathrm{Ni}-\mathrm{Ga},{ }^{11} \mathrm{Fe}-\mathrm{Ni}-\mathrm{Ga},{ }^{12}$ and $\mathrm{Ni}-\mathrm{Mn}-\mathrm{Al},{ }^{13-16}$ have been encountered.

$\mathrm{Ni}-\mathrm{Mn}-\mathrm{Ga}$ and $\mathrm{Ni}-\mathrm{Mn}-\mathrm{Al}$ are isoelectronic at equal $\mathrm{Ga}$ and $\mathrm{Al}$ concentrations when the $\mathrm{Ni}$ and $\mathrm{Mn}$ contents are kept fixed. Close to the stoichiometric Heusler composition, they are ferromagnetic (FM) in the $L 2_{1}$ parent phase and undergo a martensitic transformation at temperatures below their respective Curie temperatures $T_{C}$ exhibiting MSM properties. ${ }^{17,18}$

One also observes martensitic transformations in $\mathrm{Ni}$ Mn-Z Heusler alloys where Z can be a group III or group IV element such as In, $\mathrm{Sn}$, or $\mathrm{Sb}$, namely other than $\mathrm{Ga}$ or Al. ${ }^{19}$ In a considerable region around the stoichiometric Heusler 
TABLE I. Compositions of the $\mathrm{Ni}_{0.50} \mathrm{Mn}_{0.50-x} \mathrm{Sn}_{x}$ samples determined by EDX analysis and the valence electron concentrations per atom $e / a$ determined as the concentration-weighted sum of $s, d$, and $p$ electrons.

\begin{tabular}{ccccc}
\hline \hline$x$ & $\mathrm{Ni}$ & $\mathrm{Mn}$ & $\mathrm{Sn}$ & $e / a$ \\
\hline 0.25 & 49.6 & 24.9 & 25.5 & 7.723 \\
0.20 & 50.5 & 29.2 & 20.3 & 7.906 \\
0.18 & 49.5 & 32.3 & 18.2 & 7.939 \\
0.15 & 50.1 & 34.5 & 15.4 & 8.041 \\
0.13 & 49.9 & 37.1 & 13.0 & 8.107 \\
0.10 & 49.5 & 40.4 & 10.1 & 8.182 \\
0.05 & 49.8 & 45.1 & 5.1 & 8.341 \\
\hline \hline
\end{tabular}

composition, the structure of $\mathrm{Ni}_{2} \mathrm{MnSn}$ can be $L 2_{1}$ and $\mathrm{DO}_{3}$. The binaries $\mathrm{Ni}-\mathrm{Mn}$ and $\mathrm{Ni}$-Sn undergo martensitic transformations, whereby the product phases are tetragonal and hexagonal, respectively. As $\mathrm{Sn}$ or $\mathrm{Mn}$ is added to these binaries as the third element to form the ternary $\mathrm{Ni}-\mathrm{Mn}$-Sn, the presence of a martensitic transformation persists up to around 10 at. $\%$ substitute atom. The alloy series $\mathrm{Ni}_{0.50} \mathrm{Mn}_{0.50-x} \mathrm{Sn}_{x}$ with $0.05 \leqslant x \leqslant 0.25$ is particularly interesting in relation to structural and magnetic properties. In this work, we present a study on the structural properties and the nature of the magnetic coupling in the parent and product phases of this series. The aim is to provide an understanding of the basic properties of martensitic transformations in these alloys.

\section{EXPERIMENT}

Ingots of about $3 \mathrm{~g}$ were prepared by arc-melting the pure metals under argon atmosphere in a water-cooled $\mathrm{Cu}$ crucible. They were remelted several times. The ingots were subsequently encapsulated under argon in quartz glass and annealed at $1273 \mathrm{~K}$ for $2 \mathrm{~h}$. They were then quenched in ice water. The compositions of the $\mathrm{Ni}_{0.50} \mathrm{Mn}_{0.50-x} \mathrm{Sn}_{x}$ alloys were determined by energy dispersive X-ray analysis (EDX) and are collected in Table I. The corresponding valence electron concentration $e / a$ (electrons per atom) is also given in the table. This is calculated as the concentration weighted sum of the number of $3 d$ and $4 s$ electrons of $\mathrm{Ni}$ and $\mathrm{Mn}$ and the number of $4 s$ and $4 p$ electrons of $\mathrm{Sn}$.

Polycrystalline samples of $30-100 \mathrm{mg}$ cut from the ingots using a low speed diamond saw were used as samples for magnetization and calorimetric studies. For the differential scanning calorimetry (DSC) measurements, one side of the samples were ground down to 1200 grit $\mathrm{SiC}$ abrasive to insure proper thermal contact. Calorimetric measurements were carried out in the temperature range $100 \mathrm{~K} \leqslant T$ $\leqslant 830 \mathrm{~K}$ in a modulated DSC (TA Instruments MDSC 2920), which can be operated in both standard DSC and modulated modes. For standard DSC measurements, the cooling and heating rates were $2-5 \mathrm{~K} / \mathrm{min}$. We have also used a separate high sensitivity calorimeter to determine the entropy change of the martensitic transformation in the temperature range $100 \mathrm{~K} \leqslant T \leqslant 350 \mathrm{~K}$. Typical cooling and heating rates in these measurements were $0.5 \mathrm{~K} / \mathrm{min}$.
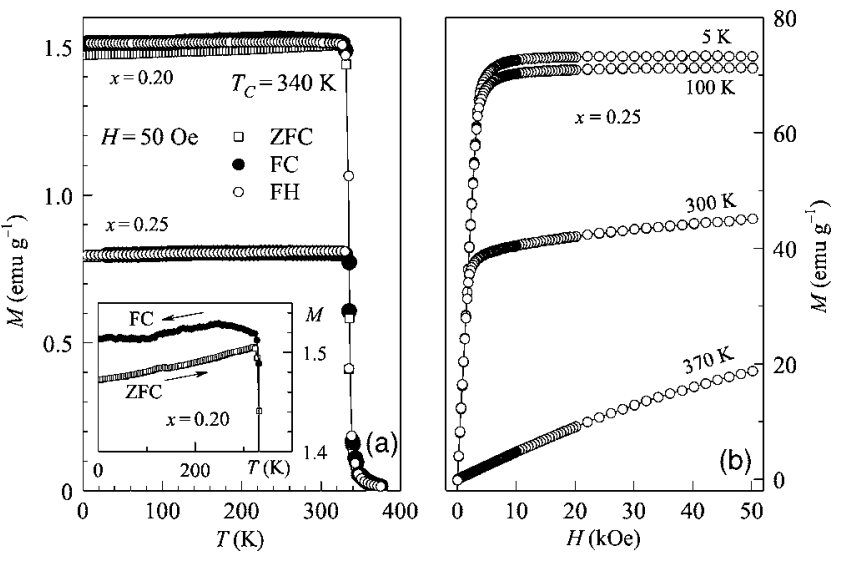

FIG. 1. (a) ZFC, FC, and FH $M(T)$ of the samples with $x$ $=0.25$ and 0.20 in $H=50$ Oe. The inset, with a higher resolution in the vertical scale, highlights the splitting of the ZFC and FC curves for $x=0.20 . M$ in the inset is in units of emu $\mathrm{g}^{1}$ (b) $M(H)$ of the sample with $x=0.25$. Prior to each $M(H)$ measurement, the samples were prepared in the ZFC state by bringing them above $380 \mathrm{~K}$.

The temperature dependence of the magnetization $M(T)$ in a low external magnetic field $(H=50 \mathrm{Oe})$ was measured in the temperature range $5 \mathrm{~K} \leqslant T \leqslant 380 \mathrm{~K}$ using a superconducting quantum interference device magnetometer. Prior to the measurements, the sample was prepared in a zero-fieldcooled state (ZFC) by cooling it from 380 to $5 \mathrm{~K}$ in the absence of a magnetic field. Subsequently, the external field was applied and the measurements were taken on increasing temperature up to $380 \mathrm{~K}$. Then, without removing the external field, the measurement was made on decreasing temperature, i.e., field-cooled (FC). As a last step, again without removing the external field the magnetization was measured on increasing temperature. The last step is denoted as the field-heated (FH) sequence. Any hysteresis in the FC-FH sequence is expected to be associated with a structural transition, whereas any splitting of the ZFC and FC curves below the ferromagnetic transition temperatures is expected to be associated with coexisting antiferromagnetic (AF) exchange. To further characterize the magnetic properties, the magnetic field dependence of the magnetization $M(H)$ was measured in fields up to $50 \mathrm{kOe}$.

Room temperature structural analysis was carried out with conventional x-ray techniques using $\mathrm{Cu} K \alpha$ radiation, and the microstructure of the specimens was examined by optical microscopy. For these studies, the samples were polished and subsequently etched in Oberhoffer solution $\left(1 \mathrm{~g} \mathrm{CuCl}_{2}, 30 \mathrm{~g}\right.$ $\mathrm{FeCl}_{3}, 0.5 \mathrm{~g} \mathrm{SnCl}_{2}, 42 \mathrm{ml} \mathrm{HCl}, 500 \mathrm{ml} \mathrm{H} \mathrm{H}_{2} \mathrm{O}$, and $500 \mathrm{ml}$ $\left.\mathrm{C}_{2} \mathrm{H}_{5} \mathrm{OH}\right)$.

\section{RESULTS}

\section{A. Magnetization studies}

$M(T)$ and $M(H)$ for all samples are presented in Figs. $1-5$. Figure 1(a) shows $M(T)$ for the sample with $x=0.20$ and for the sample with stoichiometric composition $x=0.25$. The behavior of $M(T)$ for the sample with $x=0.25$ is that of a typical ferromagnet with a Curie temperature $T_{C}=340 \mathrm{~K}$. 

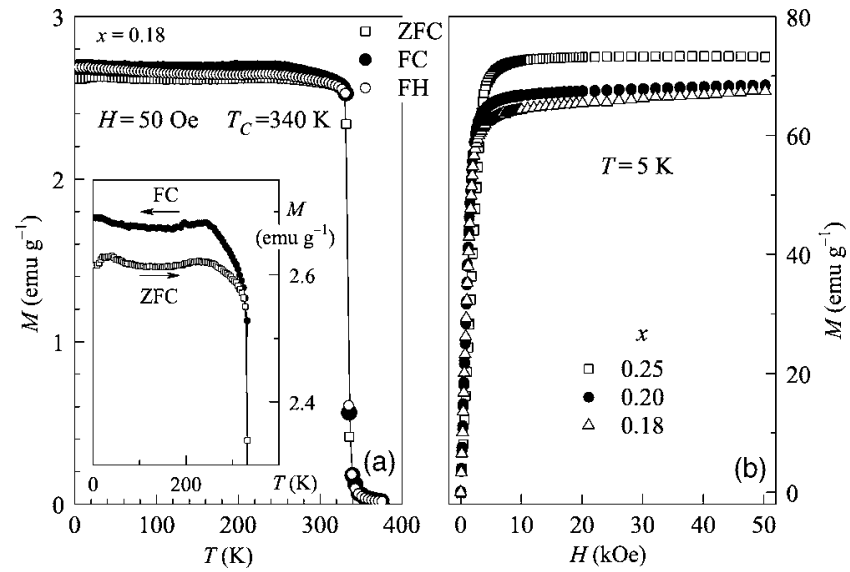

FIG. 2. (a) ZFC, FC, and FH $M(T)$ of the sample $x=0.18$ in $H=50$ Oe. The inset, with a higher resolution in the vertical scale, highlights the splitting of the ZFC and FC curves. (b) $M(H)$ at $5 \mathrm{~K}$ of the samples $x=0.25,0.20$, and 0.18 . Prior to each $M(H)$ measurement the samples were prepared in the ZFC state by bringing them above $380 \mathrm{~K}$.

Even in a low external magnetic field of 50 Oe, the FC curve retraces the ZFC curve at a magnetization value corresponding to the demagnetizing limit of the sample. The FH curve also retraces the FC and ZFC curves. $M(H)$ data at various temperatures are given in Fig. 1(b). The magnetization saturates at low temperatures, and at $300 \mathrm{~K}$ (close to $T_{C}$ ) the saturation weakens leading to a finite high field susceptibility. The $M(H)$ data at $370 \mathrm{~K}$, which corresponds to the paramagnetic state, is initially linear. However, at higher fields, the data deviate from linearity due to the presence of short range FM correlations.

As $\mathrm{Mn}$ is substituted for $\mathrm{Sn}$, excess Mn atoms occupy $\mathrm{Sn}$ sites. In such spatial configurations, $\mathrm{Mn}$ atoms can have $\mathrm{Mn}$ atoms as nearest neighbors along the [110] directions. The $\mathrm{Mn}-\mathrm{Mn}$ spacing, in this case, is smaller than that in the stoichiometric compound, and is, therefore, expected to in-
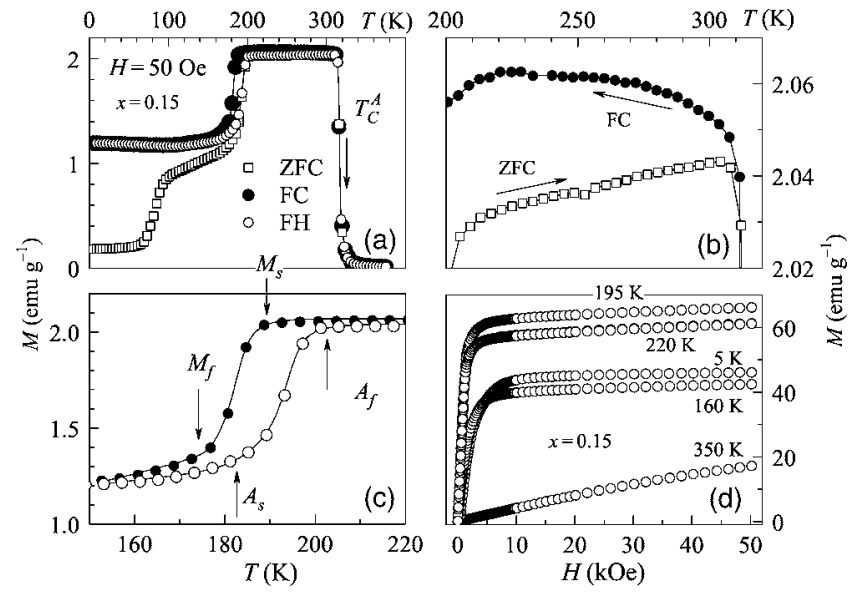

FIG. 3. (a) ZFC, FC, and FH $M(T)$ of the sample with $x=0.15$ in $H=50$ Oe. (b) $M(T)$ plotted in a temperature range around the martensitic transition. (c) $M(H)$ at 5, 160, 195, 220, and $350 \mathrm{~K}$. Prior to each $M(H)$ measurement, the samples were prepared in the ZFC state by bringing them above $380 \mathrm{~K}$.

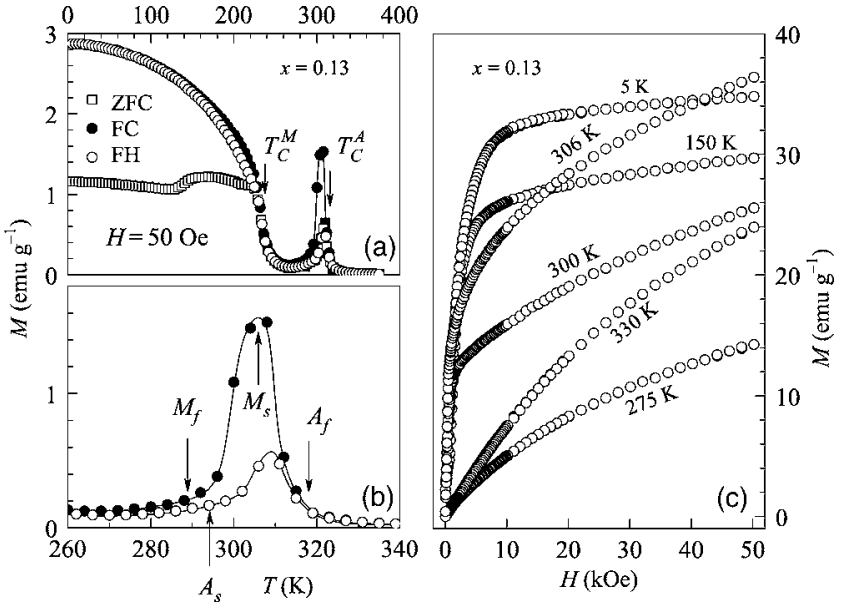

FIG. 4. (a) ZFC, FC, and FH $M(T)$ of the sample with $x=0.13$ in $H=50$ Oe. (b) $M(T)$ plotted in a temperature range around the martensitic transition. (c) $M(H)$ at 5, 150, 275, 300, 306, and $330 \mathrm{~K}$. Prior to each $M(H)$ measurement, the samples were prepared in the ZFC state by bringing them above $380 \mathrm{~K}$.

troduce $\mathrm{AF}$ exchange leading to local noncollinear spin structures, ${ }^{20,21}$ which can pin the FM domains in different configurations depending on whether the sample is cooled through $T_{C}$ in an external field or not. The different configurations cause a separation of the FC and ZFC curves just below the Curie temperature. ${ }^{22,23}$ The separation is found below $T_{C}^{A}$ for the samples in the concentration range 0.13 $\leqslant x \leqslant 0.20$. For clarity, we show this only for the samples
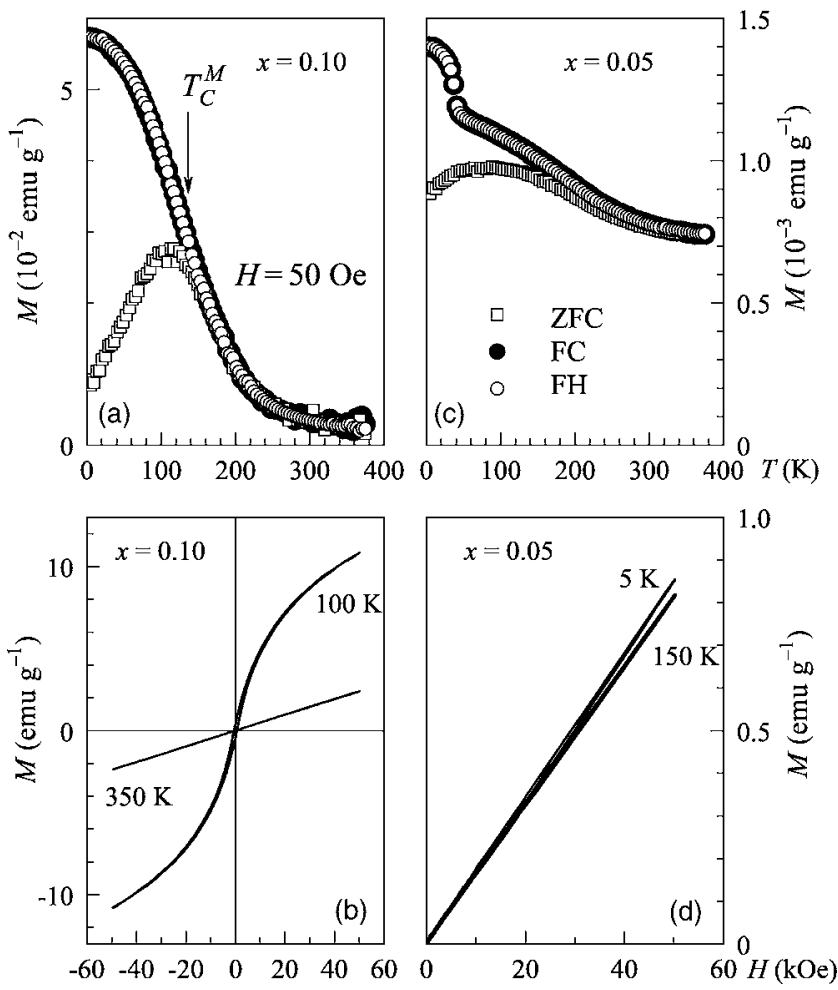

FIG. 5. (a) ZFC, FC, and FH $M(T)$ for $x=0.10$ and (b) $M(H)$ at 100 and $350 \mathrm{~K}$. (c) ZFC, FC, and FH $M(T)$ for $x=0.05$ and (d) $M(H)$ at 5 and $100 \mathrm{~K}$. 
with $x=0.20$ and 0.18 in the inset of Figs. 1(a) and 2(a). In this composition range, $T_{C}$ is nearly the same as that of the stoichiometric compound.

A further effect related to the introduction of AF exchange as the $\mathrm{Sn}$ concentration is reduced can also be identified in Fig. 2(b) where $M(H)$ at $5 \mathrm{~K}$ of the samples with $x$ $=0.18-0.25$ are compared. Whereas saturation is attained for $x=0.25$ somewhat above $15 \mathrm{kOe}, M(H)$ of $x=0.20$ and $x$ $=0.18$ do not saturate, even at $5 \mathrm{~K}$, owing to the presence of AF exchange. Furthermore, $M(H)$ in high fields decreases with decreasing Sn concentration.

As the $\mathrm{Sn}$ concentration is further decreased, we reach the situation shown in Fig. 3(a), where $M(T)$ is plotted for the sample with $x=0.15$. This sample is FM below $T_{C}^{A}=320 \mathrm{~K}$. A splitting between the ZFC and FC magnetization data [Fig. 3(b)], occurs just below $T_{C}^{A}$ as in the data for the $x=0.20$ and 0.18 samples. The ferromagnetism extends down to about $190 \mathrm{~K}$, below which the magnetization rapidly decreases. In the vicinity of this temperature, the $\mathrm{FH}$ data do not retrace the FC data but show a narrow hysteresis [Fig. 3(c)]. When considered together with the results of the calorimetric measurements presented under Sec. III B, the hysteresis can be attributed to a first-order structural transition. The forward and reverse transition temperatures have been determined by DSC measurements and are indicated with arrows. The splitting between the ZFC and FC data in the martensitic state becomes much more pronounced than that just below $T_{C}^{A}$. Evidently, the distortion of the lattice in the martensitic state has considerable effect on the pinning of the spin configurations caused by the AF exchange. As can be seen from the $M(H)$ data given at various temperatures in Fig. 3(d), the sample is in a FM state also below $M_{s}=189 \mathrm{~K}$. The magnitude of the magnetization at temperatures lower than $M_{s}$ is smaller than at higher temperatures. These points are further discussed in Sec. IV.

$M(T)$ and $M(H)$ for $x=0.13$ are plotted in Fig. 4. These data exhibit a number of features associated with a first-order structural transformation and two magnetic transitions. Here also, the structural transition temperatures are those determined from the DSC measurements [Fig. 4(b)]. At high temperatures, in the austenitic state, the sample is paramagnetic and orders ferromagnetically below $T_{C}^{A}=311 \mathrm{~K}$ [Fig. 4(a)]. The $M(H)$ curve at $330 \mathrm{~K}$ in Fig. 4(c) is linear up to about $5 \mathrm{kOe}$, after which it acquires a curvature due to the presence of ferromagnetic short range correlations- $330 \mathrm{~K}$ being close to $T_{C}^{A}$. At $306 \mathrm{~K}$, which is just below $T_{C}^{A}, M(H)$ initially rises rapidly as expected for a ferromagnet. Afterwards, the rate of increase decreases, and a saturation value is not approached. The coexistence of AF exchange within the FM matrix due to excess $\mathrm{Mn}$ in the crystal structure is the essential source for nonsaturation. The presence of AF exchange is further evidenced by the different temperature behavior of the FC and ZFC curves beginning just below $T_{C}^{A}$.

At $M_{s}=307 \mathrm{~K}$, slightly below $T_{C}^{\mathrm{A}}$, a martensitic transformation takes place. As seen in Fig. 4(a), and more detailed in Fig. 4(b), the martensitic transformation is identified by the hysteresis in the FC and FH $M(T)$ curves occurring in a narrow temperature interval just below $T_{C}^{A}$. For temperatures lower than $M_{f}, M(T)$ of the FC and FH measurement se- quences retrace. The calorimetric data presented in Sec. III B also confirm the presence for this first-order structural transition. Below $M_{s}$, the magnetization decreases with decreasing temperature as seen in Fig. 4(b). The decrease is an indication that the martensitic state does not sustain long range ferromagnetism at these temperatures. This property is also observed in $M(H)$ [Fig. 4(c)]. $M(H)$ in high external fields drops progressively as the temperature decreases from 306 to $300 \mathrm{~K}$, and finally to $275 \mathrm{~K}$. With decreasing temperature, the proportion of martensite increases, and the effect of ferromagnetic exchange of the austenite phase weakens. At $275 \mathrm{~K}$, the characteristics of the $M(H)$ data indicate that long-range ferromagnetism vanishes, and the form of the $M(H)$ curve resembles more that for $330 \mathrm{~K}$. There is no longer a rapid initial rise of $M(H)$ as for the data at $300 \mathrm{~K}$. It is not possible to determine from the present data what the exact nature of the magnetic coupling is at these temperatures. Further studies with neutron techniques would be required to understand this aspect of the problem.

As the temperature decreases, a second magnetic transition is observed at $T_{C}^{M}=230 \mathrm{~K}$, where the magnetic state of the martensitic phase becomes ferromagnetic [Fig. 4(a)]. There is no essential difference in $M(T)$ of the FC and FH states indicating that the structural transformation is essentially complete at these temperatures. On the other hand, the separation in $M(T)$ between the FC and ZFC states below $T_{C}^{M}=230 \mathrm{~K}$ is much more pronounced than that at $T_{C}^{A}$, as in the case of the sample with $x=0.15$. This shows that AF exchange persists at these temperatures, as can also be seen in the nonsaturating property of $M(H)$ at temperatures below $150 \mathrm{~K}$ shown in Fig. 4(c).

At lower Sn concentrations, long range FM ordering weakens appreciably. This can be seen in the $M(T)$ and $M(H)$ data for the samples with $x=0.10$ and 0.05 in Fig. 5 . The magnetizations of these samples in 50 Oe [Figs. 5(a) and $5(\mathrm{c})]$ are three to four orders of magnitude smaller than in the case for the samples with well-defined FM ground states. The FC $M(T)$ data for $x=0.10$ [Fig. 5(a)] exhibits a broad magnetic transition over more than $100 \mathrm{~K}$ in spite of the small external measuring field of $50 \mathrm{Oe}$. The Curie temperature of this transition is at about $137 \mathrm{~K}$; close to the temperature where the splitting occurs. The splitting of the FC and ZFC curves indicates that considerable AF exchange is present. The FM character is also evidenced by the $M(H)$ data at $100 \mathrm{~K}$ in Fig. 5(b). At temperatures above $T_{C}^{M}, M(H)$ shows a linear behavior at high temperatures, whereas below this temperature, the curve is nonlinear and exhibits a small coercivity in the hysteresis.

The values of $M(T)$ for $x=0.10$ [Fig. 5(c)] are an order of magnitude smaller than those for $x=0.10 . M(H)$ is also linear at low and high temperatures, as seen in Fig. 5(d), indicating that ferromagnetism is no longer present at this concentration. At such high Mn concentrations, one would rather expect $\mathrm{AF}$ exchange to predominate, which would account for the linearity of $M(H)$. In spite of predominating antiferromagnetism in this sample, a splitting is still found between the ZFC and FC data in Fig. 5(c) suggesting that the sample is magnetically inhomogeneous. 

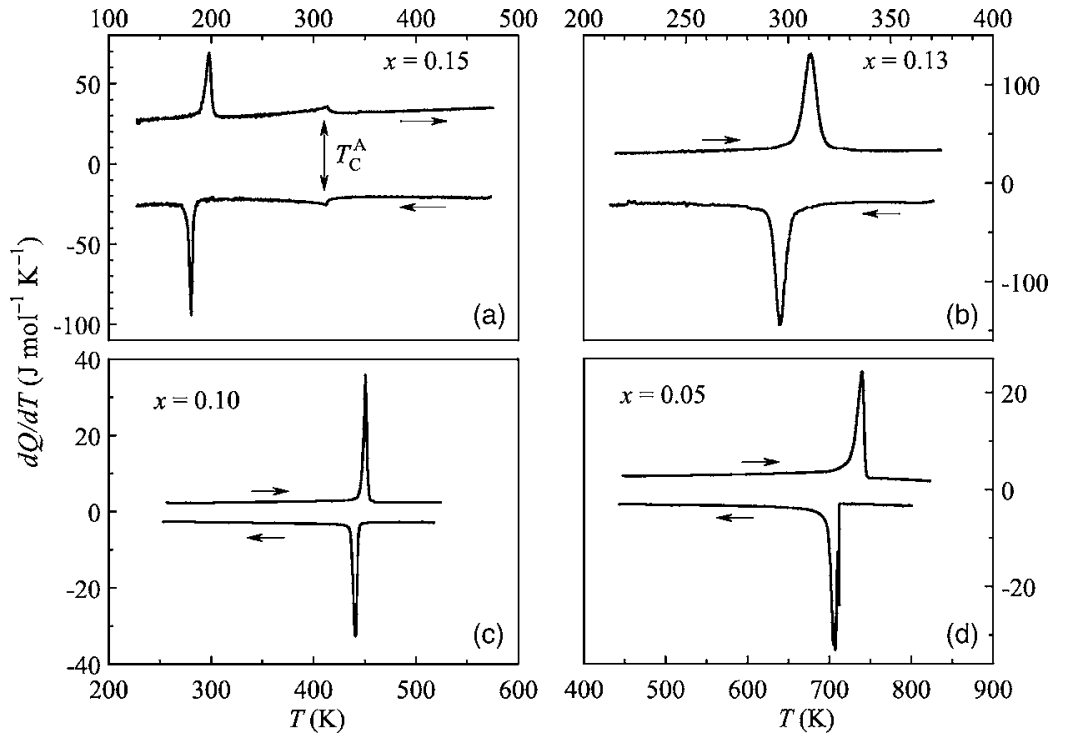

FIG. 6. $d Q / d T$ vs temperature for the alloys undergoing martensitic transformations (a) $x$ $=0.15$, (b) $x=0.13$, (c) $x=0.10$, and (d) $x=0.05$.

\section{B. Calorimetric studies}

In order to characterize the martensitic transformation in more detail, we have studied the thermal properties of $\mathrm{Ni}_{0.50} \mathrm{Mn}_{0.50-x} \mathrm{Sn}_{x}$ by DSC. For the alloys with $x \geqslant 0.18$, no structural transformation has been observed.

The results of calorimetric studies for the alloys $0.05 \leqslant x$ $\leqslant 0.15$ are plotted in Figs. 6(a)-6(d), where the heating and cooling cycles are shown by the arrows. These samples exhibit a first-order structural transformation, for which $M_{s}$ increases with decreasing $\mathrm{Sn}$ concentration. We collect in Table II the transition temperatures $M_{s}, M_{f}, A_{s}$, and $A_{f}$ and the width of the hysteresis $\Delta T$ determined as the difference between the temperatures corresponding to the peak positions. A further feature is observed at $T_{C}^{A}$ for $x=0.15$ in Fig. 6(a). This feature is not observable for $x=0.13$ because of the close-lying $T_{C}^{A}$ and $M_{s}$ temperatures. The entropy and enthalpy changes ( $\Delta S$ and $\Delta H$, respectively) around the structural transitions are calculated from the baseline corrected calorimetry data using the relationships

$$
\Delta H=\int_{T_{i}}^{T_{f}}\left(\frac{d Q}{d t}\right)\left(\frac{d T}{d t}\right)^{-1} d T
$$

and

$$
\Delta S=\int_{T_{i}}^{T_{f}} \frac{1}{T}\left(\frac{d Q}{d t}\right)\left(\frac{d T}{d t}\right)^{-1} d T,
$$

where $T_{i}$ and $T_{f}$ are the initial and final temperature limits of integration. The values of $\Delta H$ and $\Delta S$ are also included in
Table II. In the cooling experiments in Fig. 6(d), additional peaks appear in the heat flow that are related to the transformation kinetics. Various martensitic structures can be identified which are found in the x-ray spectra in the next section.

\section{Structural properties}

Figure 7 shows an x-ray diffraction pattern taken at room temperature for the alloys with $x=0.25$ and 0.15 . The superstructure reflections with the Miller indices $h, k, l=2 n+1$ are also observed implying that the patterns are related to the $L 2_{1}$ phase. By replacing $\mathrm{Sn}$ with $\mathrm{Mn}$, which has a smaller atomic radius, the lattice constants of the austenitic phase becomes smaller, and the peak positions shift to larger angles. At room temperature, the alloys with $x \leqslant 13$ are martensitic, in agreement with the calorimetric measurements. However, the structure of the martensite changes as the Mn concentration is varied as seen in Figs. 8-10. Figure 8 shows the diffraction pattern of the alloy with $x=0.13$. The reflections can be attributed to the $10 M$ modulated martensite structure. The diffraction pattern of the alloy with $x=0.10$ (Fig. 9) is related to that of a $14 M$ martensitic structure. The inset of Fig. 9 shows the details in the range $38^{\circ} \leqslant 2 \theta$ $\leqslant 46^{\circ}$. The unit cell is monoclinic making an angle $\beta$ $=93.84^{\circ}$. For $x=0.05$, the martensite has an unmodulated double tetragonal $L 1_{0}$ structure as determined from the spectrum in Fig. 10. Such structures are also observed in NiMn-Ga alloys. The results of the X-ray diffraction experiments are collected in Table III. Three of the studied alloys are in the martensitic phase at room temperature.

TABLE II. Structural transition temperatures and the associated characteristic thermal parameters.

\begin{tabular}{cccccccc}
\hline \hline$x$ & $M_{s}(\mathrm{~K})$ & $M_{f}(\mathrm{~K})$ & $A_{s}(\mathrm{~K})$ & $A_{f}(\mathrm{~K})$ & $\Delta T(\mathrm{~K})$ & $\Delta H\left(\mathrm{~J} \mathrm{~mol}^{-1}\right)$ & $\Delta S\left(\mathrm{~J} \mathrm{~mol}^{-1} \mathrm{~K}^{-1}\right)$ \\
\hline 0.15 & 189 & 174 & 190 & 202 & 19 & $313 \pm 53$ & $1.66 \pm 0.13$ \\
0.13 & 307 & 289 & 295 & 318 & 19 & $911 \pm 65$ & $3.0 \pm 0.3$ \\
0.10 & 444 & 437 & 445 & 453 & 10 & $1642 \pm 63$ & $3.7 \pm 0.2$ \\
0.05 & 711 & 693 & 718 & 743 & 31 & $3060 \pm 360$ & $4.3 \pm 0.7$ \\
\hline \hline
\end{tabular}




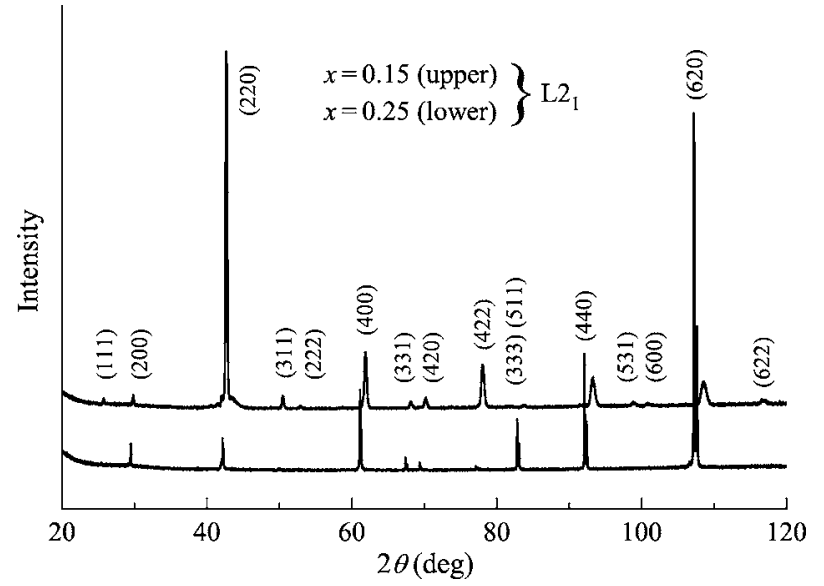

FIG. 7. X-ray diffraction pattern at room temperature for $x$ $=0.25$ (lower) and $x=0.15$ (upper). The crystal structure is in both cases $L 2_{1}$.

The variation of the crystal structure at room temperature from $L 2_{1}$ to the modulated structures $10 M$, to $14 M$, and then to $L 1_{0}$, as the $\mathrm{Sn}$ concentration decreases, can also be seen in the microstructure of the alloys shown in Figs. 11(a)-11(d). These are optical microscopy images of the surface of the alloys with $x=0.05,0.10,0.13$, and 0.25 . Figure 11 (a) shows the surface of the sample with $x=0.25$, for which the grain size varies in the range $50-300 \mu \mathrm{m}$. The black spots are voids caused by the etching during surface preparation.

Figures 11(b)-11(d) show the microstructure of the samples that are martensitic at room temperature. No phase separation can be identified. This is also in agreement with the x-ray diffraction patterns, where all reflections are related to the martensitic phases. The grain size lies within the range 50-300 $\mu \mathrm{m}$. The morphologies of the $10 M, 14 M$, and the unmodulated phases appear to be different from one another. Although in all three cases the martensite is platelike, which can be recognized by the linear grain boundary of each plate, the widths of the twins are different for $10 M, 14 M$, and $L 1_{0}$. For $10 M$ martensite, a broad form is found, whereas for $14 M$ martensite, the form is finer, and that of the unmodulated

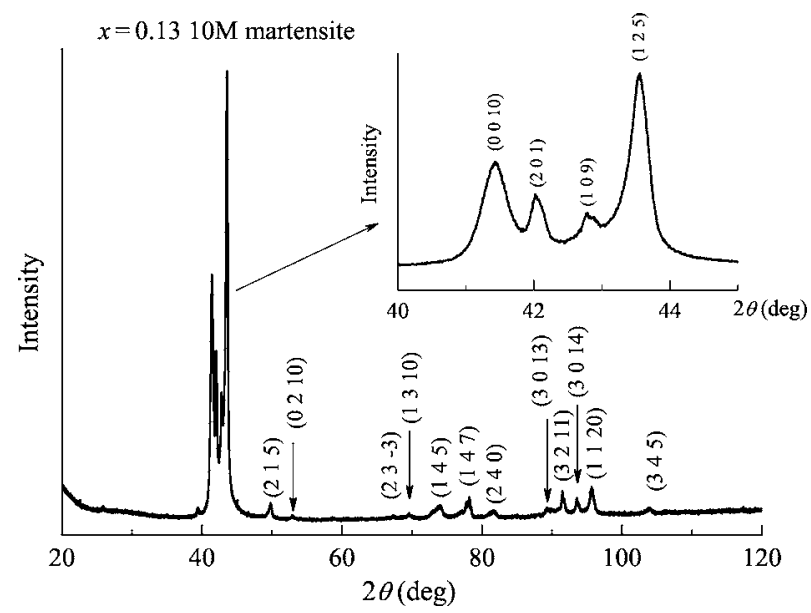

FIG. 8. X-ray diffraction pattern at room temperature for $x$ $=0.13$. The crystal structure is $10 M$ with an orthorhombic unit cell.

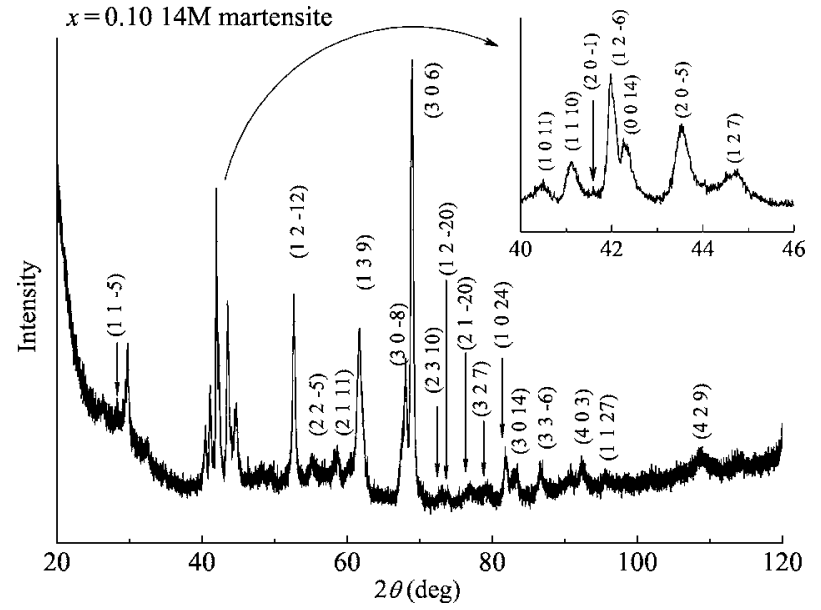

FIG. 9. X-ray diffraction pattern at room temperature for $x$ $=0.10$. The inset shows details in the range $40^{\circ} \leqslant 2 \theta \leqslant 46^{\circ}$. The crystal structure is $14 M$ with a monoclinic unit cell.

structure is in between. Within the grains, the plates order parallel as seen in Figs. 11(b) and 11(c). For the case of the $L 1_{0}$ structure, the plates are not ordered.

\section{DISCUSSION}

In the $M(T)$ data of the sample with $x=0.13$, two distinct FM states with $T_{C}^{M}<T_{C}^{A}$ for the martensitic and austenitic phases can be observed. No long range ferromagnetism is present in the temperature range $T_{C}^{M}<T<T_{C}^{A}$.

Two distinct FM states are also found for $x=0.15$ that can be evidenced in the $M(H)$ data in Fig. 3(c). In this figure, it is seen that below $T_{C}^{A}, M(H)$ saturates above about $15 \mathrm{kOe}$. However, the saturation magnetizations are smaller in the range $T<M_{s}$ (5 and $\left.160 \mathrm{~K}\right)$ than for $T>M_{s}$ (195 and $220 \mathrm{~K})$. This is seen in more detail in Fig. 12, where $M(T)$ in high magnetic fields of $50 \mathrm{kOe}$ is plotted. Starting from low temperatures, $M(T)$ decreases with increasing temperature and conforms to a $T^{3 / 2}$ Bloch law. The inset shows a linear behavior of the $T^{3 / 2}$ dependence of the reduced magnetiza-

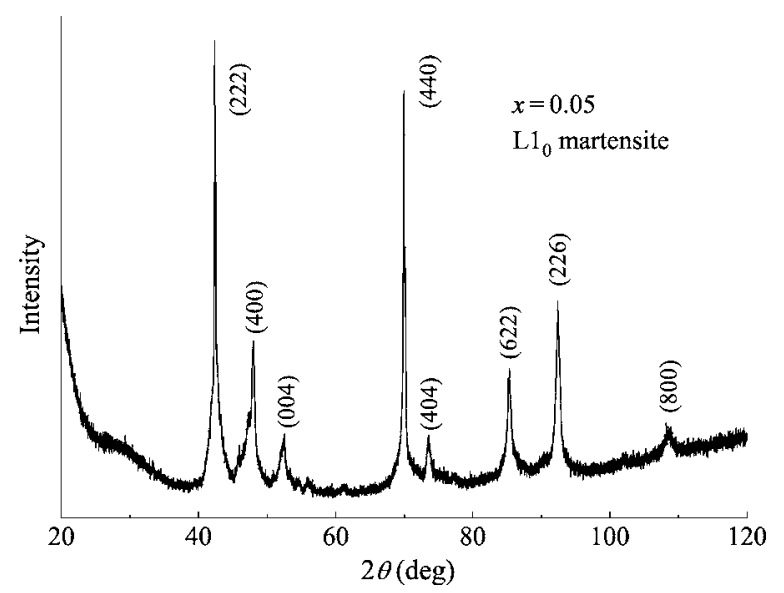

FIG. 10. X-ray diffraction pattern at room temperature for $x$ $=0.10$. The crystal structure is $L 1_{0}$. 
TABLE III. Crystal structures and lattice parameters at room temperature of the samples. The structure of the sample with $x$ $=0.10$ is monoclinic with $\beta=93.84^{\circ}$.

\begin{tabular}{|c|c|c|c|c|}
\hline \multirow[b]{2}{*}{$x$} & \multirow[b]{2}{*}{$\begin{array}{l}\text { Crystal } \\
\text { structure }\end{array}$} & \multicolumn{3}{|c|}{ Lattice parameter } \\
\hline & & $a(\AA)$ & $b(\AA)$ & $c(\AA)$ \\
\hline 0.25 & $L 2_{1}$ & 6.046 & 6.046 & 6.046 \\
\hline 0.20 & $L 2_{1}$ & 6.024 & 6.024 & 6.024 \\
\hline 0.18 & $L 2_{1}$ & 6.009 & 6.009 & 6.009 \\
\hline 0.15 & $L 2_{1}$ & 5.995 & 5.995 & 5.995 \\
\hline 0.13 & $10 M$ & 4.317 & 5.621 & 21.808 \\
\hline 0.10 & $14 M$ & 4.333 & 5.570 & 29.971 \\
\hline 0.05 & $L 1$ & 7.595 & 7.595 & 6.980 \\
\hline
\end{tabular}

tion $M / M_{0}$, where $M_{0}$ is taken as the magnetization at $T$ $=5 \mathrm{~K}$. Since Bloch behavior is valid essentially for $T$ $<T_{C} / 3, T_{C}^{M}$ is expected to be virtual and lie around $600 \mathrm{~K}$, i.e., at a temperature higher than $T_{C}^{A}$. This property is also found to be consistent with the concentration dependence of the structural and magnetic phase transitions discussed below. The dashed line in Fig. 12 is a guide that represents the behavior of $M(T)$ beyond the stability range of the martensitic state.

A similar effect showing different magnetic exchange in austenitic and martensitic states is encountered also in $\mathrm{Ni}$ Mn-Ga alloys. ${ }^{24,25}$ However, in these alloys, the ferromagnetic state in the martensitic phase has a higher magnetization than in the austenitic phase, and $T_{C}^{M}$ is always greater than $T_{C}^{A}$.

A feature that particularly stands out in the $M(T)$ data of the $x=0.15$ and 0.13 samples is the pronounced increase in the splitting between the ZFC and FC $M(T)$ curves in the FM martensitic state as opposed to the weak splitting in the FM austenitic state. It is not possible from the present data to give an exact account for such a large scale increase. The rearrangement of the antiphase boundaries caused by the twinning in the distorted martensitic state can have an influence on the Mn-Mn interatomic spacings so as to cause an enhancement in the strength of the AF exchange. A closer study of the martensitic structure is needed to clarify this issue.

In the concentration range $8.0 \leqslant e / a \leqslant 8.2$, the $\mathrm{Ni}_{0.50} \mathrm{Mn}_{0.50-x} \mathrm{Sn}_{x}$ system features diverse structural and magnetic transitions as seen in the phase diagram in Fig. 13. Data from literature are also included in the diagram. ${ }^{19,21} T_{C}^{A}$ decreases slightly with increasing $e / a$ up to $e / a \simeq 8$.1. Above this concentration, the system is in the martensitic state at room temperature, and the Curie temperature, now $T_{C}^{M}$, decreases rapidly with increasing $e / a$. This sudden change in the $e / a$ dependence of the Curie temperature is attributed to a change in the ferromagnetic exchange mechanism in the martensitic phase with respect to that in the austenitic state. The crystallographic distortion in the martensitic state evidently leads to an alteration in the band splitting of the electronic structure so as to cause a considerable change in the FM ordering temperature. Using our present data and those
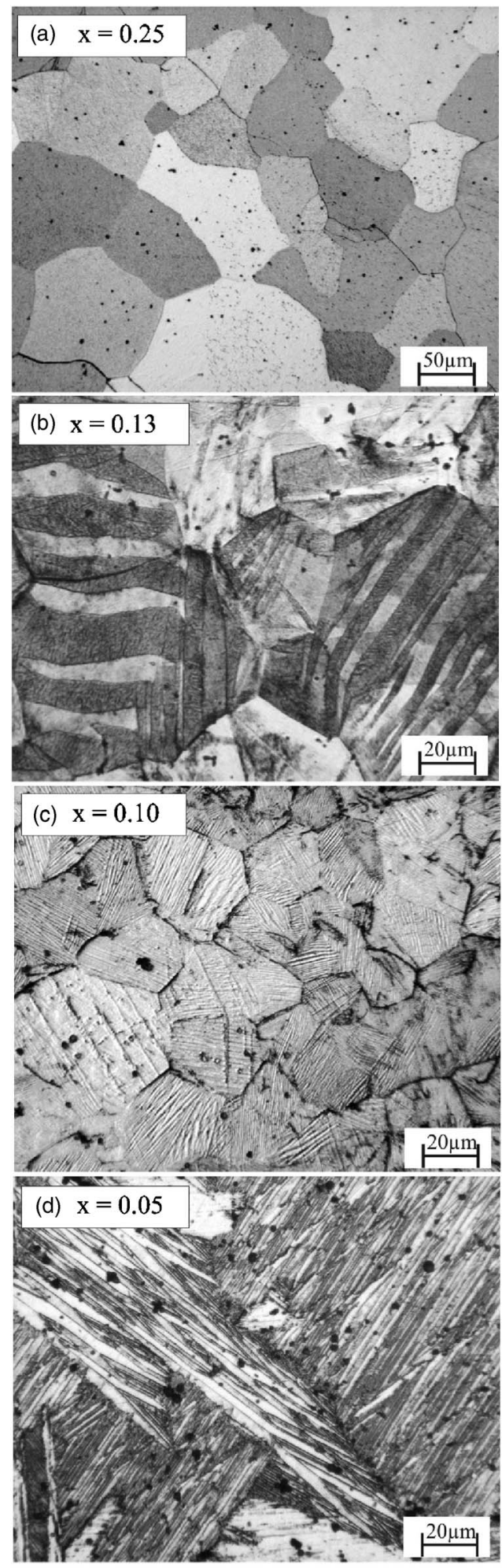

FIG. 11. Optical microscopy images of the microstructure for $x=0.25\left(L 2_{1}\right), x=0.13(10 M), x=0.10(14 M)$, and $x=0.05\left(L 1_{0}\right)$.

of Ref. 19, we incorporate $T_{C}^{M}$ into the phase diagram. $T_{C}^{M}$ is expected to vanish at about $e / a=8.2(x=0.10)$. If the line describing $T_{C}^{M}$ is extrapolated back to smaller $e / a$ values, one finds that for about $x<0.14, T_{C}^{M}>T_{C}^{A}$. This provides support for the validity of the argument given above for the values of 


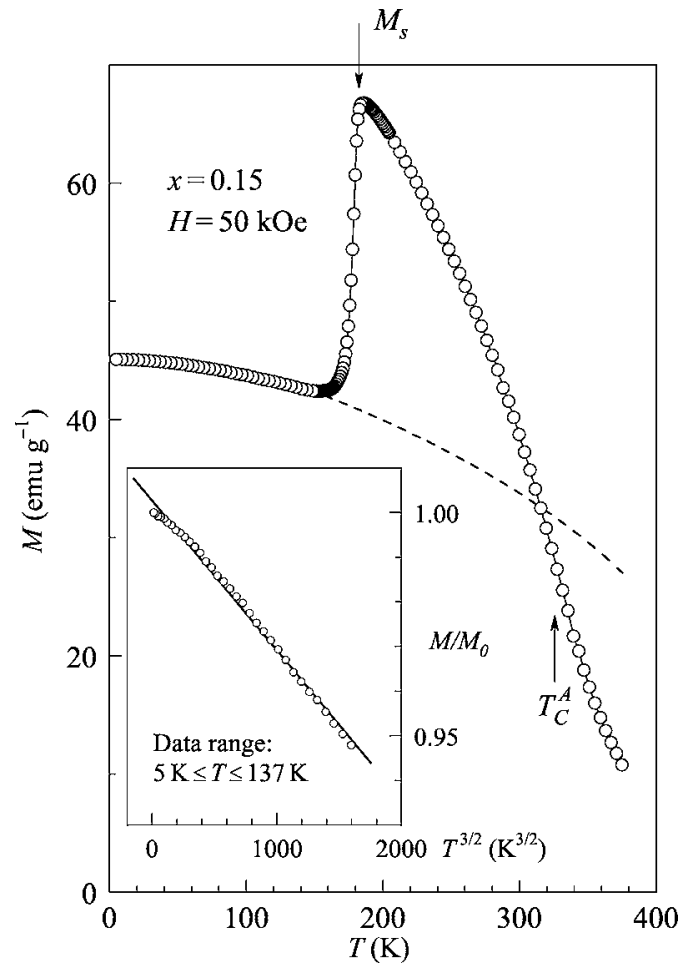

FIG. 12. $M(T)$ measured in $50 \mathrm{kOe}$. The dashed line is a guide showing the expected behavior of $M(T)$ of the martensitic state beyond the stability range. The position of $M_{s}$ indicated in the figure is taken from the results of DSC measurements. The inset shows the linear behavior of $M / M_{0}$ vs $T^{3 / 2}$.

the Curie temperatures of the austenitic and martensitic states for $x=0.15$.

As the Sn concentration decreases, FM exchange weakens, and long range ferromagnetism no longer exists below

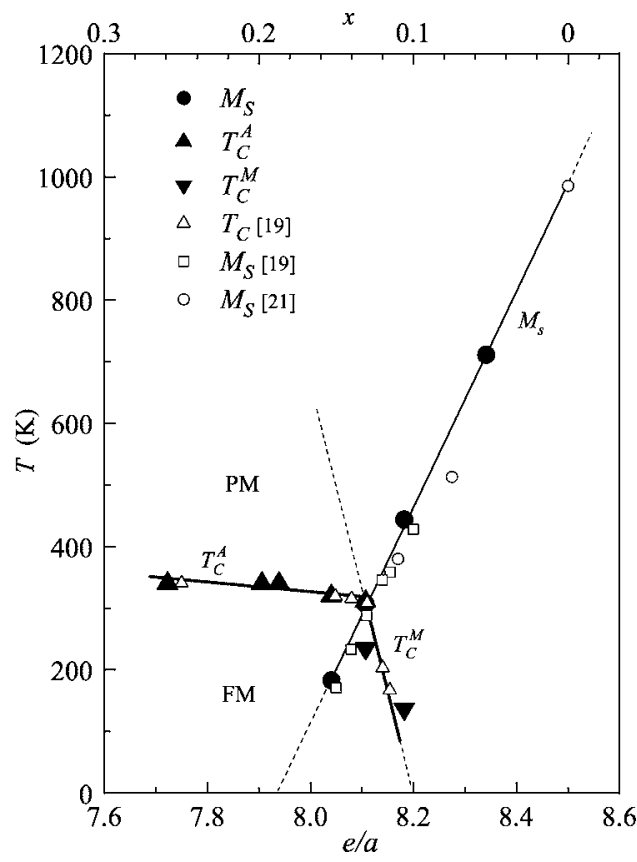

FIG. 13. The structural and magnetic transition temperatures as a function of the valence electron concentration for $\mathrm{Ni}-\mathrm{Mn}-\mathrm{Sn}$.

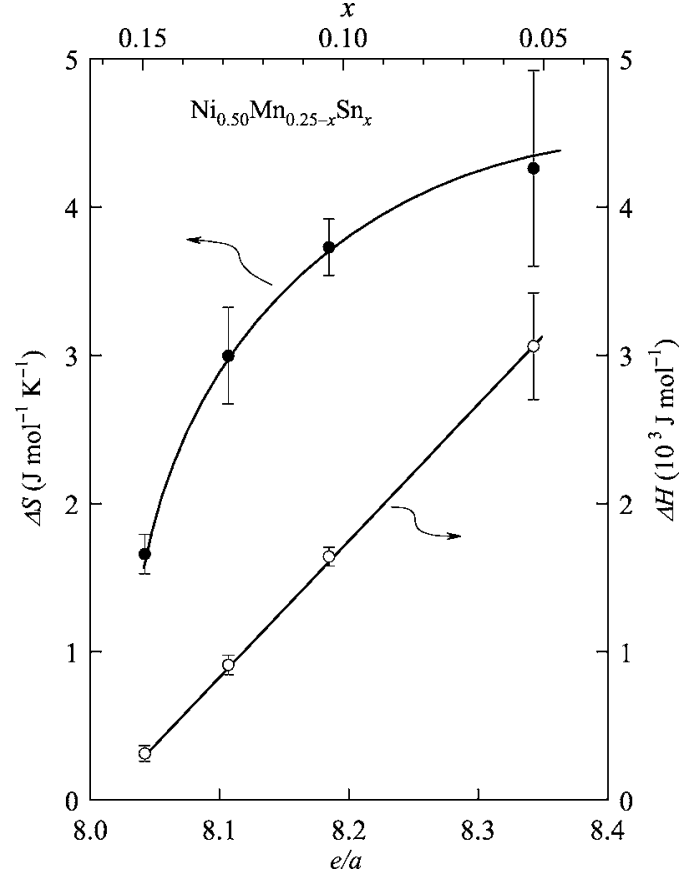

FIG. 14. $\Delta S$ and $\Delta H$ as a function of $e / a$.

about $x=0.10$. At lower Mn concentrations, the linear behavior of $M(H)$ for $x=0.05$ up to the highest magnetic fields shows that AF exchange begins to dominate. However, a Néel temperature cannot be identified in $M(T)$ in the measured temperature range.

The enthalpy and entropy of the martensitic transition increase in absolute value with increasing $e / a$ (Fig. 14). The increase in $\Delta H$ is linear, while $\Delta S$ shows a tendency to acquire a curvature at large $e / a$. The error bars are essentially related to the uncertainties in positioning the base line of $d Q / d T$ in Fig. 6. An increase of $\Delta S$ with increasing $e / a$ has also been reported for $\mathrm{Ni}-\mathrm{Mn}$ - Ga alloys. ${ }^{26}$ This behavior is in contrast to that observed in Cu-based alloys ${ }^{27}$ for which no significant $e / a$ dependence of $\Delta S$ is observed. For the Cu-based alloys, $\Delta S$ has mainly a vibrational contribution, which is related to the low-lying transverse $\mathrm{TA}_{2}$ phonon branch. It has been argued that for $\mathrm{Ni}-\mathrm{Mn}-\mathrm{Ga}$, the electron contribution to $\Delta S$ is negligible and that the character of the $e / a$ dependence is related to a magnetic contribution that relies on the difference in the magnetic exchange below and above $M_{s}$. The vibrational contribution was assumed to be independent of $e / a$. Similar arguments could also apply for $\mathrm{Ni}-\mathrm{Mn}-\mathrm{Sn}$. In fact, in the case of Ni-Mn-Sn, the difference in the magnetic exchange interactions below and above $M_{s}$ is much larger than in Ni-Mn-Ga, ${ }^{28}$ as seen in Figs. 3 and 4. Such a difference would account for the larger values found in $\mathrm{Ni}-\mathrm{Mn}-\mathrm{Sn}$ when compared to those reported for $\mathrm{Ni}$ $\mathrm{Mn}-\mathrm{Ga}$. A detailed investigation aimed at separating the different contributions to the entropy change in magnetic shape memory alloys would sustain the above arguments.

\section{CONCLUSION}

Optical microscopy and $\mathrm{x}$-ray studies show that the type of modulated structure of the martensite in $\mathrm{Ni}_{0.50} \mathrm{Mn}_{0.50-x} \mathrm{Sn}_{x}$ 
depends on the Sn composition. As the Sn concentration increases, the structure varies in a sequence $10 M, 14 M$, and $L 1_{0}$. In the event that the austenitic and martensitic phases are FM, the ferromagnetism of the parent and product phases are found to be of a different nature, whereby each is characterized by a separate magnetic transition temperature. The presence of ferromagnetism in the martensitic state is necessary for a magnetic shape memory effect to occur and is found in the alloys with $x=0.13$ and 0.15 . However, whether any magnetic shape memory effect occurs in these alloys is yet to be demonstrated. The present study will be complimented by magnetostriction studies to address this question.

\section{ACKNOWLEDGMENTS}

We thank Peter Hinkel and Sabine Schremmer for technical support. This work was supported by Deutsche Forschungsgemeinschaft (GK277) and CICyT (Spain), Project No. MAT2004-1291. X.M. acknowledges support from DGICyT (Spain).
*Electronic address: macet@agfarle.uni-duisburg.de

${ }^{1}$ L. C. Chang and T. A. Read, Trans. AIME 191, 47 (1951).

${ }^{2}$ Shape Memory Materials, edited by K. Otsuka and C. M. Wayman (Cambridge University Press, Cambridge, England, 1998).

${ }^{3}$ K. Ullakko, J. K. Huang, C. Kantner, R. C. O'Handley, and V. V. Kokorin, Appl. Phys. Lett. 69, 1966 (1996).

${ }^{4}$ K. Ullakko, J. K. Huang, V. V. Kokorin, and R. C. O'Handley, Scr. Mater. 36, 1133 (1997).

${ }^{5}$ R. C. O’Handley, J. Appl. Phys. 83, 3263 (1998).

${ }^{6}$ R. C. O'Handley, S. J. Murray, M. Marioni, H. Nembach, and S. M. Allen, J. Appl. Phys. 87, 4712 (2000).

${ }^{7}$ J. Pons, V. A. Chernenko, R. Santamarta, and E. Cesari, Acta Mater. 48, 3027 (2000)

${ }^{8}$ T. Kakeshita, T. Takeuchi, T. Fukuda, T. Saburi, R. Oshima, S. Muto, and M. Kishio, Mater. Trans., JIM 41, 882 (2000).

${ }^{9}$ R. D. James and M. Wuttig, Philos. Mag. A 77, 1273 (1998).

${ }^{10}$ K. Oikawa, L. Wulff, T. Iijima, F. Gejima, T. Ohmori, A. Fujita, K. Fukamichi, and K. Ishida, Appl. Phys. Lett. 79, 3290 (2001).

${ }^{11}$ K. Oikawa, T. Ota, F. Gejima, T. Ohmori, R. Kainuma, and K. Ishida, Mater. Trans., JIM 42, 2472 (2001).

${ }^{12}$ K. Oikawa, T. Ota, T. Ohmori, H. Morito, A. Fujita, R. Kainuma, K. Fukamichi, and K. Ishida, Appl. Phys. Lett. 81, 5201 (2002).

${ }^{13}$ R. Kainuma, H. Nakano, and K. Ishida, Metall. Mater. Trans. A 27, 4153 (1996).

${ }^{14}$ S. Morito and K. Otsuka, Mater. Sci. Eng., A 208, 47 (1996).

${ }^{15}$ A. Fujita, K. Fukamichi, F. Gejima, R. Kainuma, and K. Ishida,
Appl. Phys. Lett. 77, 3054 (2000).

${ }^{16}$ M. Acet, E. Duman, E. F. Wassermann, L. Mañosa, and A. Planes, J. Appl. Phys. 92, 3867 (2002).

${ }^{17}$ L. Mañosa, A. Planes, M. Acet, E. Duman, and E. F. Wassermann, J. Appl. Phys. 93, 8498 (2003).

${ }^{18}$ L. Mañosa, A. Planes, M. Acet, E. Duman, and E. F. Wassermann, J. Magn. Magn. Mater. 272-276, 2090 (2004).

${ }^{19}$ Y. Sutou, Y. Imano, N. Koeda, T. Omori, R. Kainuma, K. Ishida, and K. Oikawa, Appl. Phys. Lett. 85, 4358 (2004).

${ }^{20}$ C. V. Stager and C. C. M. Campbell, Can. J. Phys. 56, 674 (1978).

${ }^{21}$ E. Wachtel, F. Henninger, and B. Predel, J. Magn. Magn. Mater. 38, 305 (1983).

${ }^{22}$ E. Duman, M. Acet, Y. Elerman, A. Elmali, and E. F. Wassermann, J. Magn. Magn. Mater. 238, 11 (2002).

${ }^{23}$ I. Nowik, I. Felner, and E. R. Bauminger, Phys. Rev. B 55, 3033 (1997).

${ }^{24}$ V. V. Khovailo, V. Novosad, T. Takagi, D. A. Filippov, R. Z. Levitin, and A. N. Vasil'ev, Phys. Rev. B 70, 174413 (2004).

${ }^{25}$ R. Tickle and R. D. James, J. Magn. Magn. Mater. 195, 627 (1999).

${ }^{26}$ V. V. Khovailo, K. Oikawa, T. Abe, and T. Tagaki, J. Appl. Phys. 93, 8483 (2003).

${ }^{27}$ A. Planes and Ll. Mañosa, Solid State Phys. 55, 159 (2001).

${ }^{28}$ J. Marcos, A. Planes, L. Mañosa, F. Casanova, X. Batlle, A. Labarta, and B. Martínez, Phys. Rev. B 66, 224413 (2002). 